\title{
Quantitative or Qualitative Research: A Futile Dispute
}

\section{Ezatolah Naderi* and Maryam Seifnaraghi}

Department of Educational Sciences, Science and Research Branch, Deakin University, Australia

*Corresponding author: Ezatolah Naderi, Department of Educational Sciences, Science and Research Branch, Deakin University, Australia, Tel: +61392446100; Email: eznaderi@hotmail.com

Rec date: September 13, 2017; Acc date: November 03, 2017; Pub date: November 06, 2017

Copyright: @ 2017 Naderi E, et al. This is an open-access article distributed under the terms of the creative commons attribution license, which permits unrestricted use, distribution, and reproduction in any medium, provided the original author and source are credited.

\section{Abstract}

This article seeks to decrease discrepancies on the disputes about quantitative, qualitative, and mixed method research, using clear explanations and drawing on well-evidenced examples. All in the hope that it might do justice to a better understanding of the act of research as the sole approach available to any scholar for the protection of their preferentiality and authoritativeness.

Keywords: Quantitative; Qualitative; Human sciences; Accidents Scientific research

\section{Introduction}

Recently, one can see many disputes and controversies over quantitative and qualitative research methods in articles on research. Some scholars try to see quantitative research special to hard sciences only, while some others consider qualitative research appropriate solely to human sciences; so much so that they regard a researcher in qualitative research a "player" but a "spectator" in quantitative research [1].

\section{Literature Review}

Some others maintain that making use of mathematics and statistics in research "quantitativism" and see this type of thinking as positivism, thus attaching some inappropriateness and improperness to it [2]. Therefore, if attempt is not made at understanding mathematical concepts and statistics as a result, we will probably have no way but fabricate myths even in everyday affairs. For instance, if we do not estimate the speed with which a vehicle might safely run on a zigzag road with many hair-pin bends, it is very likely that we attribute accidents caused by centrifugal force to some evil or ghosts.

In order to settle the current disputes, some commentators have proposed the concept of "mixed method approach to research" in recent years. In some cases, they even write: "...regarding research methods, attention to man's move from unorganized methods to quantitative ones, from quantitative to qualitative ones, and finally resort to mix method approach is of high importance" [3]. Some colleagues also assert: "... gone through a relatively rapid growth spurt... it has acquired a formal methodology that did not exist before and is subscribed to by an emerging community of practitioners and methodologists across the disciplines. In the process of developing a distinct identity, as compared with other major research communities of researchers in the social and human sciences, mixed methods have been adopted as the de facto third alternative, or third methodological movement [4]."

It should be asserted that all such inferences and opinions emanate from an insufficient understanding of the true nature of "scientific research". According to what has come to be accepted as "research" in the course of history, the concept is nothing but "search for truth and attaining to deep down of issues". On the other hand, for noble and free men who don't have access to the occult, but do have some knowledge of a discipline, searching for truth is possible only through resorting to pure wisdom [5] and that with some limitation; that is, they will be able to get close to some parts or particular manifestations of truth. Put differently, it is evident that in order to sustain and pursue their authoritativeness, any scholar or intellectual having some knowledge of limited scope, will have to avail of carrying out research in order to find answers to questions or solutions to problems on the way of their growth and development.

\section{Discussion}

Therefore, research-the correct way of attaining truth-is any scholar's occupation; and the generalities of doing so has been the same for all disciplines in the course of history; only the subject of exploration differs. This is because, as it was noted, the way of research enjoys universal features of pure wisdom, and as a "mixed activity" consists of a series of activities each complementing the other. Research in any field of knowledge, for instance, starts where the agent of exploration comes to acknowledge their ignorance on the trajectory to wisdom [6].

In their first step after the first spark, a researcher has to search within their own knowledge bounds, and come to perceive the subject in question within the reaches of their current knowledge. In other words, they have to start from the "review of literature" or "background of the topic" or alternatively the "theoretical framework" of the research topic. This type of study on the research topic bears many advantages with it, including the fact that it helps the researcher elaborate on the research objectives, hypotheses, and questions bearing in mind integration with the research topic literature and attending to possibilities they have access to. After carrying out this step, and gaining an adequate perception of the research topic known as "statement of the problem"-where they have to clearly and with simplicity express distinguishing features of the research variables as well as reasons for studying them-the researcher has to justify the significance and necessity of the study with well-evidenced reasons, considering the selected research method, research context or statistical population, and at the same time, identifying the sample size-explaining why and how they have decided over it-beforehand, or 
Citation: Naderi E, Seifnaraghi M (2017) Quantitative or Qualitative Research: A Futile Dispute. J Trauma Treat 6: 401. doi:

Page 2 of 2

alternatively postponing it after data collection process is complete and adequate data are at hand to the point of saturation.

Consequently, the researcher must reflect upon the collected data in line with investigating objectives, testing hypotheses, or responding questions, making use of the available sample prepared either way. In terms of the data used in research, heed has to be paid to the following points:

- No research is conceivable without data. In some research, data to help with answering questions or testing hypotheses or investigating the research objectives, are not already there; a researcher therefore must generate or develop them, as it is done in experimental research.

- In some other research, to answer questions, achieve goals, or test hypotheses, the needed data do exist; a researcher therefore must only adopt and/or collect them. In yet another group of research, a researcher encounters a set of previously stored data, he has had no role for collection of; including what is stored in storage and backup memories in supermarkets or organizations every day, week, month, etc.

It is now evident that a researcher must adopt a method or approach in order to organize or array the data collected using any of the above ways. This method is the very way(s) by which man has been able to achieve goals such as cost reduction, acceleration, accuracy enhancement, and extending different phenomena's scope of understanding and recognition; and introduce concepts such as organization, order, sorting out, large or small, preceding or following logically; and inventing numbers and finally exercise some operations on numbers and figures known as "mathematics", a branch of which is statistics.

\section{Conclusion}

On this account, it can be seen that research as the sole avenue at any researcher's avail for protection and maintenance of their "referentiality" and authoritativeness, has from the very beginning been mixed and blended an action; something the execution of which enjoys clear rules such as "systematicity", planning and arrangement, accuracy, patience, fairness, conciseness, and "professionality" at a universal scope. Additionally, it is congruent with man's pure nature and wisdom, not bound to geographical borders in history and thus has been and will continue to be acceptable. Now, if such rules are not observed in the course of carrying out research as a result of negligence, carelessness, or individual desires or biases, the quality of research, and in turn, its product quality-science-will be at stake; the consequences of which will be nothing but culture and economic losses.

\section{References}

1. Bagheri KH (2010) Research approaches and methods in philosophy of education, Research Institute for Cultural and Social Studies, Ministry of Higher Education, Tehran, Iran.

2. Reich Y (2009) Educational innovations quarterly. "Special issue on research methodology", Research Institute for Curriculum Planning and Educational Innovations, Tehran, Iran.

3. Soroush A (2001) The creation resort (5th edn); Seraat Cultural Institute, Tehran, Iran.

4. Naderi E, Seifnaraghi M (2010) Shortcomings and pitfalls in research: A categorization; Arasbaran Publications, Tehran, Iran.

5. Naderi E, Seifnaraghi M (2015) Research Methods and its Evaluation in Human Sciences; Arasbaran Publications, Tehran, Iran.

6. Tashakkori A, Teddlie C (2010) "Epilogue: Current developments and emerging trends in integrated research methodology", In: Sage Handbook of Mixed Methods in Social \& Behavioral Research (Eds) 2010, Sage, California, USA. pp. 803-826. 